\title{
Enzyme-Instructed Activation of Pro-protein Therapeutics (PPT) In Vivo
}

Jin Chang ${ }^{\dagger, \star}$, Weiqi Cai ${ }^{\dagger \dagger}$, Chunjing Liang ${ }^{\dagger, \ddagger}$, Qiao Tang ${ }^{\dagger} \star$, Xianghan Chen ${ }^{\dagger, \star}$, Ying Jiang ${ }^{\S}$, Lanqun Mao ${ }^{\dagger, \star}$, Ming

Wang $\dagger$ †**

$\dagger$ Beijing National Laboratory for Molecular Sciences, Key Laboratory of Analytical Chemistry for Living Biosystems, Institute of Chemistry, Chinese Academy of Sciences (CAS), Beijing 100190, China

$\ddagger$ University of Chinese Academy of Sciences, Beijing 100049, China

$\S$ College of Chemistry, Beijing Normal University, Beijing 100875, China

Email Address: mingwang@iccas.ac.cn 


\section{Experimental Section:}

General All reagents for chemical synthesis were purchased from commercial sources and used as received. Recombinant green fluorescent protein (GFP) was expressed in E. Coli. using the pET28b:GFP plasmid obtained from Addgene (\#60733). Dicoumarol and bovine pancreatic ribonuclease A (RNase A) were purchased from Sigma-Aldrich (St. Louis, MO). Cytochrome c was purchased from Solarbio (Beijing, China). NAD(P)H: quinone oxidoreductase 1 (NQO1), and 2,3-dimethoxy 1,4-naphthoquinone (DMNQ) were purchased from Abcam (Boston, USA). 2-(4-iodophenyl)-3-(4-nitrophenyl)- 5-(2,4-disulfophenyl)-2H-tetrazolium (WST-1) was purchased from BioVision (Milpitas, USA). Circular dichroism spectra were recorded using Jasco J-815 Circular Dichroism (CD) Spectropolarimeter (Jasco, Japan).

\section{Synthesis of QPN and PN ligand}<smiles>CC1=C(C)C(=O)C(C(C)(C)CC(=O)O)=C(C)C1=O</smiles>

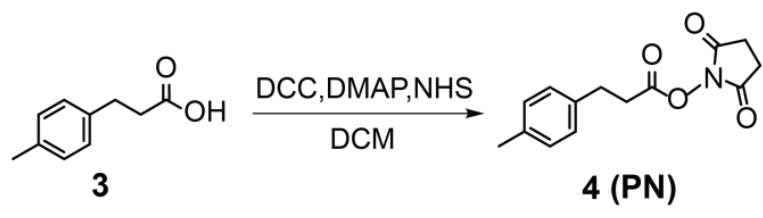

Scheme 1 Synthesis route of QPN and PN ligand for protein modifications.

Synthesis of 3-methyl-3-(2,4,5-trimethyl-3,6-dioxocyclohexa-1,4-dien-1-yl)butanoic acid(QPN). N-Hydroxysuccinimide (NHS) (0.5 g, $4.34 \mathrm{mmol})$ and 4-(N,N-dimethylamino)pyridine $(0.05 \mathrm{mg}, 0.41 \mathrm{mmol})$ were added to a solution of $\mathbf{1}(1 \mathrm{~g}$, $3.99 \mathrm{mmol})$ in dichloromethane $(35 \mathrm{~mL})$ with stirring at room temperature. $\mathrm{N}, \mathrm{N}$ '-dicyclohexylcarbodiimide $(0.8 \mathrm{~g}, 4.02$ mmol) was slowly added into the above mixture. After $7 \mathrm{~h}$, the solution was filtrated and evaporated under reduced pressure, and the remaining solution was dissolved in ice ethyl acetate, filtrated and evaporated under reduced pressure. The obtained residue was purified by column chromatography (petroleum ether and ethyl acetate, 2:1, v/v) to give QPN 
as a yellow solid (1.2 g, 90\%). ${ }^{1} \mathrm{H}$ NMR (300 MHz, Chloroform-d) $\delta 3.28(\mathrm{~s}, 2 \mathrm{H}), 2.78$ (s, 4H), 2.17 (s, 3H), $1.96(\mathrm{~s}, 6 \mathrm{H})$, 1.53 (s, 6H). MS (ESI): calcd. For $\mathrm{C}_{18} \mathrm{H}_{21} \mathrm{NO}_{6}[\mathrm{M}+\mathrm{H}]^{+} \mathrm{m} / \mathrm{z}=348.14$, Found 348.18 .

Synthesis of 3-methyl-3-(2,4,5-trimethyl-3,6-dioxocyclohexa-1,4-dien-1-yl)butanoic acid(PN). N-Hydroxysuccinimide (NHS) $(0.8 \mathrm{~g}, 6.69 \mathrm{mmol})$ and 4-(N,N-dimethylamino)pyridine $(0.05 \mathrm{mg}, 0.41 \mathrm{mmol})$ were added to a solution of 4-Methylbenzenebutanoic acid $(\mathbf{3}, 1 \mathrm{~g}, 6.09 \mathrm{mmol})$ in dichloromethane $(35 \mathrm{~mL})$ with stirring at room temperature. $\mathrm{N}, \mathrm{N}^{\prime}$-dicyclohexylcarbodiimide $(1.5 \mathrm{~g}, 7.31 \mathrm{mmol})$ was slowly added into the above mixture. After $7 \mathrm{~h}$, the solution was filtrated and evaporated under reduced pressure, and the remaining solution was dissolved in ice ethyl acetate, filtrated and evaporated under reduced pressure. The obtained residue was purified by column chromatography (petroleum ether and ethyl acetate, $3: 1, \mathrm{v} / \mathrm{v})$ to give $\mathrm{PN}$ as a yellow solid product $(1.4 \mathrm{~g}, 87 \%) .{ }^{1} \mathrm{H}$ NMR (300 MHz, Chloroform-d) $\delta 7.31$ $(\mathrm{t}, 2 \mathrm{H}), 6.93(\mathrm{t}, 2 \mathrm{H}), 2.83(\mathrm{t}, 2 \mathrm{H}), 2.78(\mathrm{~m}, 4 \mathrm{H}), 2.62(\mathrm{t}, 2 \mathrm{H}), 2.15(\mathrm{~s}, 3 \mathrm{H}) . \mathrm{MS}(\mathrm{ESI})$ : calcd. For $\mathrm{C}_{14} \mathrm{H}_{15} \mathrm{NO}_{4}[\mathrm{M}+\mathrm{H}]^{+} \mathrm{m} / \mathrm{z}=$ 262.10, Found 262.15.

General protocol for protein modification. The chemical modification of proteins was performed by mixing the protein (GFP, Cytochrome c or RNase A) (1 mg) with QPN or PN at a ligand to protein molar ratio of $300: 1$ in $1 \mathrm{~mL} \mathrm{NaHCO}_{3}$ buffer solution $(0.1 \mathrm{M}, \mathrm{pH}=9.5)$. The reaction mixture was stirred for $6 \mathrm{~h}$ at room temperature, then purified using Amicon Ultra Centrifugal Filters (MWCO 3KD) following the manufacturers' instruction. The as-obtained protein conjugates were characterized using MALDI-TOF mass spectrometry.

NQO1-catalyzed QPN removal and protein activity regulation. To demonstrate the effectiveness of NQO1 on controlling protein modification and activity, $50 \mu \mathrm{g} / \mathrm{mL}$ QPN or PN-modified proteins (GFP, Cytochrome c or RNase A) were incubated with NQO1 (100 ng/mL) along with NADH (300 mM) in Dulbecco's Phosphate Buffered Saline (DPBS) at $37{ }^{\circ} \mathrm{C}$ for $6 \mathrm{~h}$. At the end of the incubation, the reaction mixture was purified using Amicon Ultra Centrifugal Filters (MWCO 3KD), and the purified proteins were analyzed using MALDI-TOF mass spectrometry. 
To study the effect of NQO1 treatment on controlling the activity of QPN or PN-modified protein, Cyt c-QPN or Cyt c-PN $(50 \mu \mathrm{g} / \mathrm{mL})$ with NQO1 $(100 \mathrm{ng} / \mathrm{mL})$ was subjected to enzyme activity assay by mixing the protein with $25 \mathrm{mM}$ $\mathrm{H}_{2} \mathrm{O}_{2}$ and $10 \mathrm{mM}$ ABTS, followed by absorption measurement at $418 \mathrm{~nm}$. As regard the activity assay of RNase A, the NQO1-treated protein was mixed with RNase Alert kit (IDT, USA) according to our previous report for RNase A activity assay. ${ }^{1}$ To study the inhibitory effect of dicoumarol on NQO1 promoted protein activation, dicoumarol $(200 \mu \mathrm{M})$ was added to the above reaction mixture composed of QPN modified protein and NQO1 before enzyme activity assay. As regard the selective RNase A-QPN activation by NQO1, RNase A-QPN (50 $\mu \mathrm{g} / \mathrm{mL})$ was incubated with NQO1 (100 $\mathrm{ng} / \mathrm{mL})$, nitroreductase $(50 \mu \mathrm{g} / \mathrm{mL})$, bovine serum albumin $(50 \mu \mathrm{g} / \mathrm{mL})$, or fetal bovine serum $(50 \mu \mathrm{g} / \mathrm{mL})$ in DPBS for 6 h, followed by RNase A activity assay as described above.

Intracellular Delivery of RNase A-QPN using lipid nanoparticles. The library of lipid nanoparticles was synthesized and purified according to our recent report. ${ }^{2}$ To screen effective lipid nanoparticles for RNase A-QPN delivery, the lipid and protein were mixed in phosphate buffer solution $(25 \mathrm{mM}, \mathrm{pH}=7.2)$ at a weight ratio of $3: 2$; the as -prepared lipid and protein nanocomplex was added to HeLa cells seeded in a 96-well plate at a RNase A-QPN concentration of $8 \mu \mathrm{g} / \mathrm{mL}$, and lipid concentration of $12 \mu \mathrm{g} / \mathrm{mL}$, followed by cell viability measurement using MTT assay $48 \mathrm{~h}$ post delivery.

To compare the different activation of RNase A-QPN and RNase A-PN in living cells, HeLa cells were treated with different concentrations of ROS-TK-12/RNase A-QPN or ROS-TK-12/RNase A-PN nanoparticles, with the concentration of protein added to cells increasing from $0.7 \mu \mathrm{g} / \mathrm{mL}$ to $8 \mu \mathrm{g} / \mathrm{mL}$, followed by cell viability assay as described above.

NQO1 silencing inhibited RNase A-QPN activation in living cells. To knock down the cellular NQO1 expression and study its inhibitory effect on RNase A-QPN activation, HeLa cells seeded in 48-well plate at a density of 80,000 cells per well were transfected with $80 \mathrm{nM}$ siNQO1 (antisense-strand: 5'-CCGUACACAGAUACCUUGA-3') or scramble siRNA (antisense-strand: 5'-GAACUUCAGGGUCAGCUUG-3') using Lipofectamine 2000 according to the manufacturers' instructions. Cell lysates were used for intracellular NQO1 activity assay $48 \mathrm{~h}$ post siRNA transfection. To measure 
cellular NQO1 activity, cell lysates (normalized to protein concentration) were added to a mixed solution containing 10 $\mu \mathrm{M}$ WST-1, $400 \mu \mathrm{M}$ NADH, and $10 \mu \mathrm{M}$ DMNQ, followed by the absorption measurement at $450 \mathrm{~nm}$. To study the effect of NQO1 knock down on the decreased cytotoxicity of ROS-TK-12/RNase A-QPN nanoparticles, HeLa cells were transfected with siNQO1 or scramble siRNA and further treated with ROS-TK-12/RNase A-QPN nanoparticles at a protein concentration of $6.4 \mu \mathrm{g} / \mathrm{mL}$, followed by cell viability measurement and compared to that of cells without any treatment.

NOQ1 upreglation in HEK293T cells enhanced RNase A-QPN activation. Briefly, HEK293T cells seeded in 48-well plate at a density of 80,000 cells per well were treated with $1.33 \mu \mathrm{M}$ sulforaphane for $12 \mathrm{~h}$, then lysed for NQO1 activity assay as described above. To verify the enhanced RNase A-QPN activation in NQO1 up-regulated cells, HEK293T cells pre-treated with $1.33 \mu \mathrm{M}$ sulforaphane were treated with ROS-TK-12/RNase A-QPN nanoparticles at a protein concentration of $3.2 \mu \mathrm{g} / \mathrm{mL}$. Cell viability was measured and compared to that of cells without sulforaphane pre-treatment.

Intracellular delivery of RNase A-QPN for targeted cancer therapy. To demonstrate the potential of NQO1-catalyzed RNase A-QPN activation to selectively inhibit tumor cell growth, three non-cancerous cell lines, such as HEK293T, HK-2 and Swiss-3T3 cells, and three cancerous cells, such as HeLa, MCF-7, and SiHa cells were seeded in a 96-well plate at a density of $1.0 \times 10^{4}$ cells per well. These cells were treated with ROS-TK-12/RNase A-QPN nanoparticles at a protein concentration of $6.4 \mu \mathrm{g} / \mathrm{mL}$. Cell viability was measured and compared using MTT assay $48 \mathrm{~h}$ post protein delivery. To compare the different endogenous NQO1 concentration in the above cells, $25 \mathrm{ng} / \mathrm{mL}$ of cell lysates (normalized to protein concentration) were analyzed for NQO1 activity according to the method described above.

Fluorescent labeling of RNase A-QPN for in vivo study. To study the in vivo distribution of ROS-TK-12/RNase A-QPN nanoparticle using fluorescence imaging, the protein was labeled with fluorescein isothiocyanate (FITC) before use. Briefly, 2 mg RNase A-QPN dissolved in $750 \mu \mathrm{L}$ sodium bicarbonate solution $(0.1 \mathrm{M}, \mathrm{pH}=9.5)$ was mixed with 250 
$\mu \mathrm{L}$ freshly prepared FITC solution $(4 \mathrm{mg} / \mathrm{mL}$ in DMSO). The reaction mixture was then stirred at room temperature for 2 $\mathrm{h}$ in the dark. The resulted FITC-labeled RNase A-QPN was purified by size exclusion chromatography on a PD-10 desalting column (GE Healthcare, MA, USA).

In vivo RNase A-QPN delivery and anticancer efficacy. To develop HeLa tumor bearing xenograft, $6 \times 10^{6} \mathrm{HeLa}$ cells suspended in $200 \mu \mathrm{L}$ DPBS were subcutaneously injected in the left axilla region of four-week old female BALB/c (nu/nu) nude mice (purchased from Beijing Vital River Laboratory Animal Technology Co., Ltd). As regard in vivo distribution study of ROS-TK-12/FITC-RNase A-QPN nanoparticles, HeLa tumor bearing mice with a tumor size of approximately $1200 \mathrm{~mm}^{3}$ were intravenously injected with $200 \mu \mathrm{L}$ ROS-TK-12/FITC-RNase A-QPN, or free FITC-RNase A-QPN protein at a protein dosage of $2.5 \mathrm{mg} / \mathrm{kg}$. Mice were sacrificed $6 \mathrm{~h}$ post injections, and the organs of each different group were collected for fluorescence imaging on a IVIS small animal imaging system (PerkinElmer, USA).

As regard the in vivo delivery and tumor growth suppression study, mice with a tumor size of approximately 100 $\mathrm{mm}^{3}$ were intravenously injected with DPBS, ROS-TK-12/RNase A-QPN nanoparticles, ROS-TK-12 nanoparticles, or free RNase A-QPN once for every two days, five injections in total for each mouse at a protein dosage of $2.5 \mathrm{mg} / \mathrm{kg}$ and/or a ROS-TK-12 dosage of $8 \mathrm{mg} / \mathrm{kg}$ each mouse. Tumor size and mouse body weight were measured every two days. The tumor volume was calculated using the following formula: $1 / 2 \mathrm{x}$ length $(\mathrm{mm}) \times$ width $^{2}(\mathrm{~mm})$, and normalized to that of mouse before nanoparticle injection. 


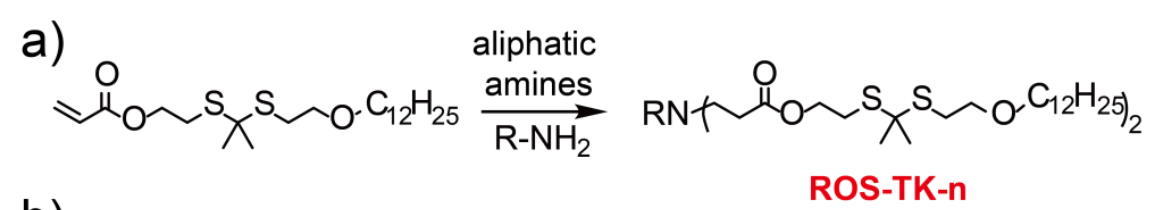

b)

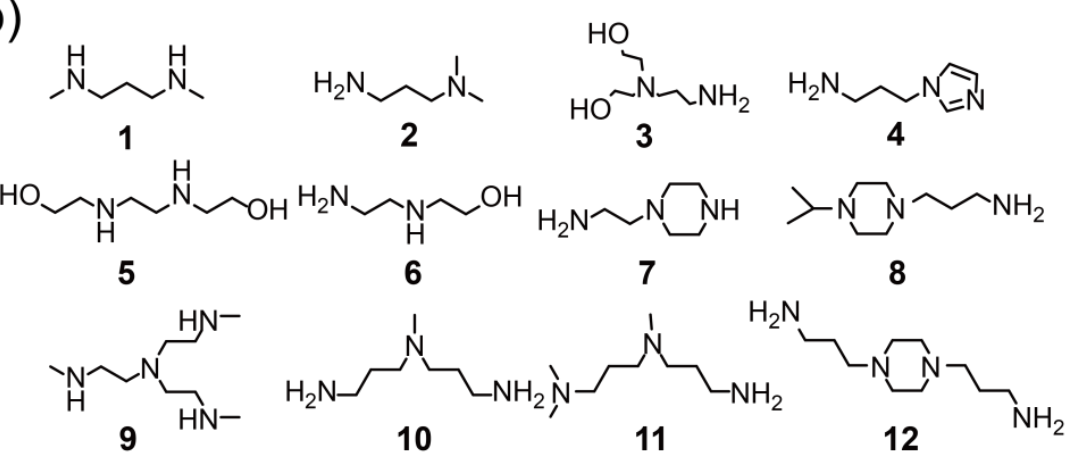

Scheme S2 a) Synthesis of the ROS-responsive lipids used for protein delivery; b) Chemical structure of aliphatic amines used to generate the library of cationic lipids in this study. 

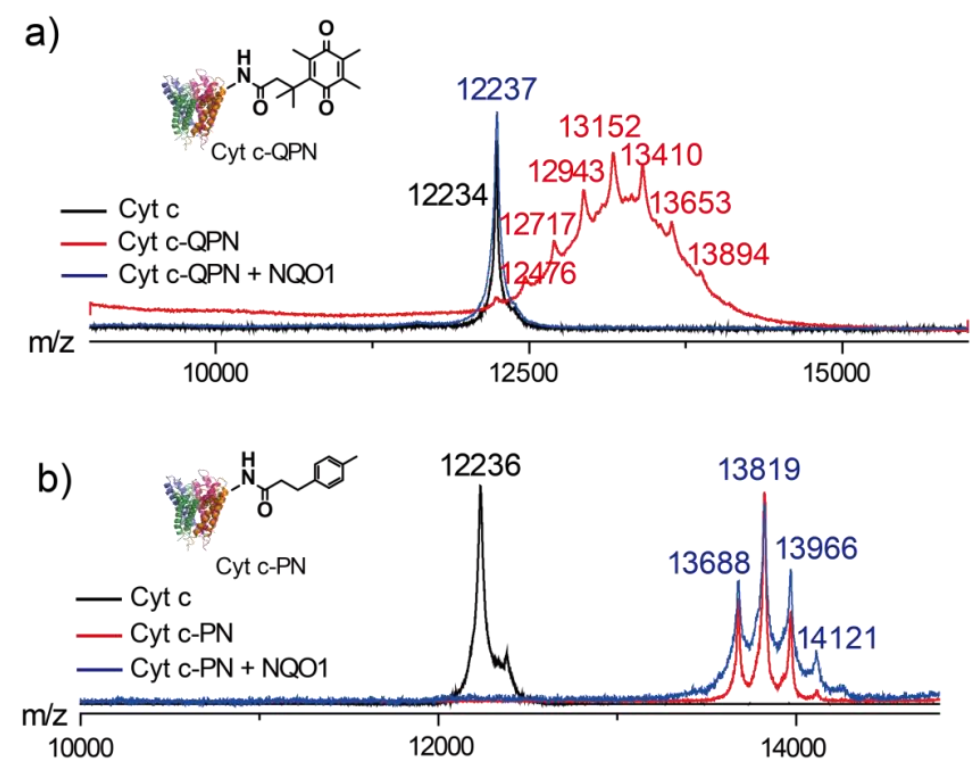

Figure S1 MALDI-TOF mass spectrometry characterization of Cyt c-QPN (a) and Cyt c-PN (b) before and after NQO1 treatment. Cyt c-QPN or Cyt c-PN $(50 \mu \mathrm{g} / \mathrm{mL})$ was treated with $100 \mathrm{ng} / \mathrm{mL}$ NQO1 before MALDI-TOF analysis. 
a)

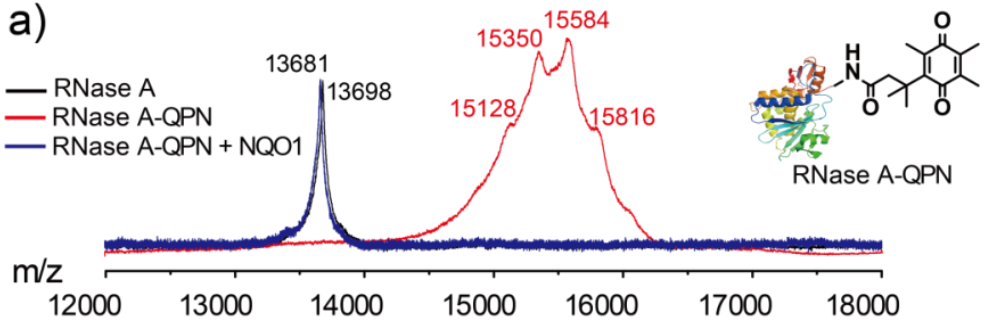

b)

$15012 \quad 15168$

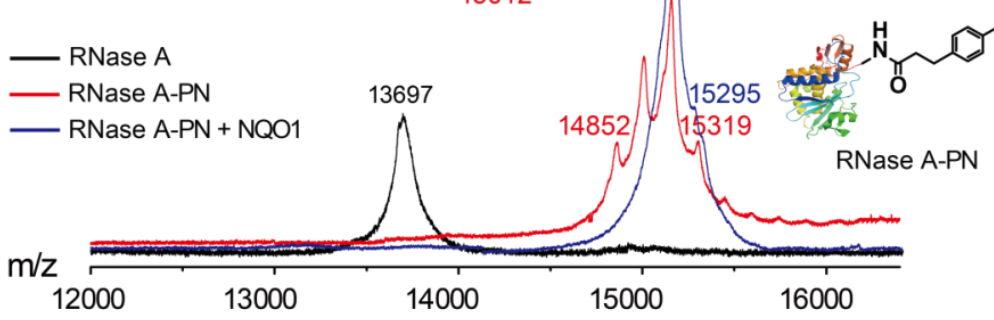

Figure S2 MALDI-TOF mass spectrometry characterization of RNase A-QPN (a) and RNase A-PN (b) before and after NQO1

treatment. RNase A-QPN or RNase A-PN $(50 \mu \mathrm{g} / \mathrm{mL})$ was treated with $100 \mathrm{ng} / \mathrm{mLNQO} 1$ before MALDI-TOF analysis.

$\begin{array}{lllll}10 & 20 & 30 & 40 & 50\end{array}$

MALKSLVLLS LLVLVLLLVR VQPSLGKETA AAKFERQHMD SSTSAASSSN

$\begin{array}{lllll}60 & 70 & 80 & 90 & 100\end{array}$

YCNQMMKSRN LTKDRCKPVN TFVHESLADV QAVCSQKNVA CKNGQTNCYQ

$\begin{array}{lllll}110 & 120 & 130 & 140 & 150\end{array}$

SYSTMSITDC RETGSSKYPN CAYKTTQANK HIIVACEGNP YVPVHFDASV

Amino acid sequence of RNase $A$

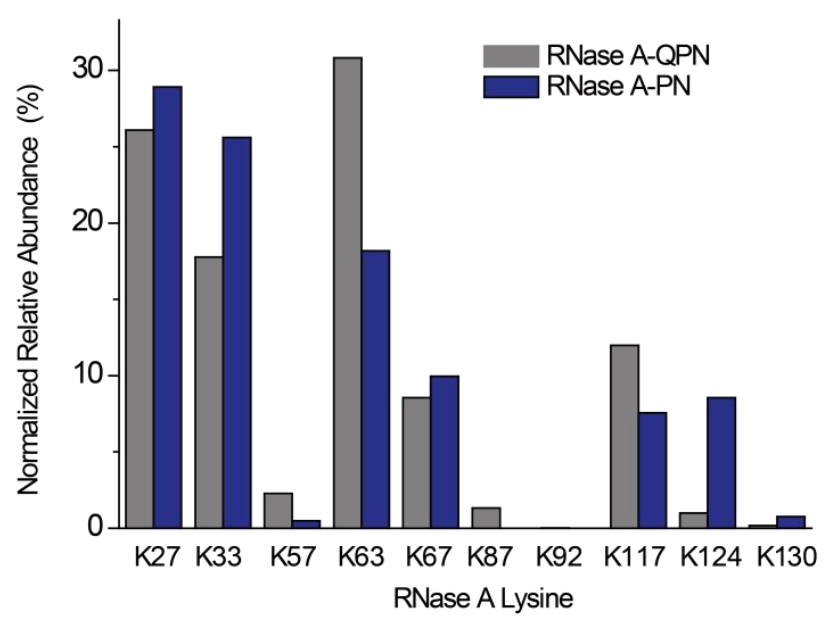


Figure S3 Amino acid sequence of RNase A and the lysine modification site of RNase A-QPN and RNase A-PN determined by mass spectrometry analysis. Proteins were digested with trypsin or chymotrypsin into peptide fragments before mass spectrometry analysis performed on a Exactive mass spectrometer (Thermo Fisher, USA)

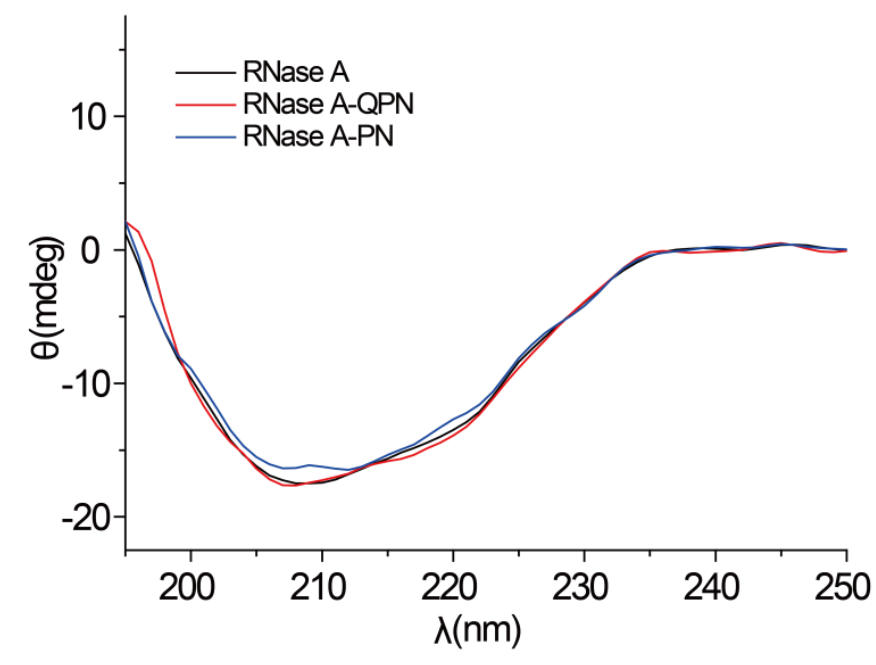

Figure S4 Circular dich roism spectra of RNase A $(0.86 \mathrm{mg} / \mathrm{mL})$ or RNase A-QPN, or RNase A-PN at the same concentration, in $10 \mathrm{mM}$ Tris (trishydroxymethylaminomethane), $\mathrm{pH}=7.6$. 


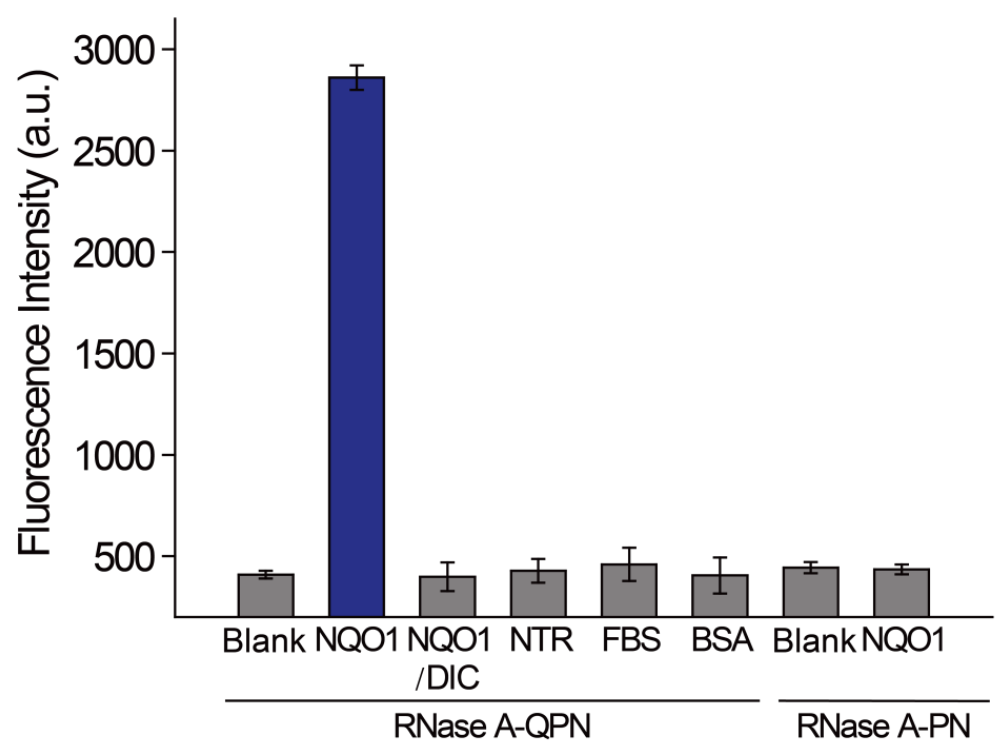

Figure S5 Selective RNase A-QPN activation by NQO1. RNase A-QPN ( $50 \mu \mathrm{g} / \mathrm{mL})$ was incubated with $100 \mathrm{ng} / \mathrm{mL}$ NQO1, or $50 \mu \mathrm{g} / \mathrm{mL}$ of NTR, FBS, or BSA for $6 \mathrm{~h}$ before enzyme activity assay. RNase A-PN was similarly treated with NQO 1 as a negative control. Results are presented as mean $\pm \mathrm{SD}$ of three independent experiments. 


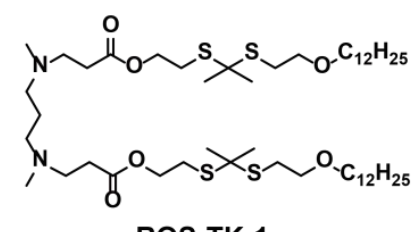

ROS-TK-1

等

ROS-TK-4

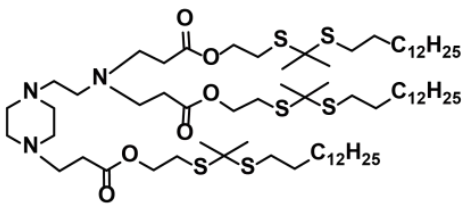

ROS-TK-7

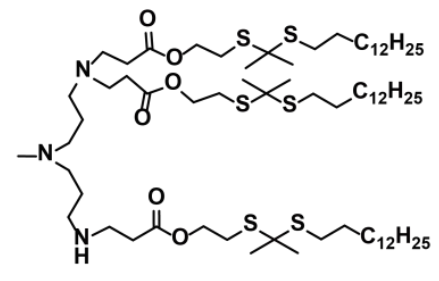

ROS-TK-10

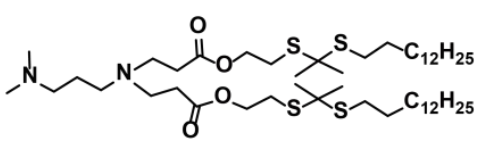

ROS-TK-2

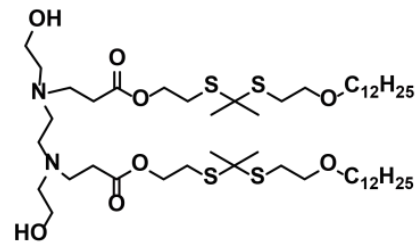

ROS-TK-5

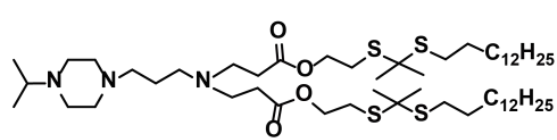

ROS-TK-8

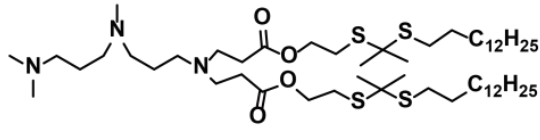

ROS-TK-11

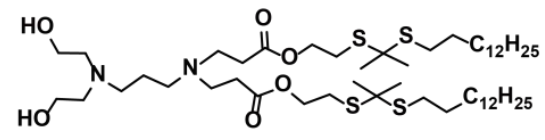

ROS-TK-3

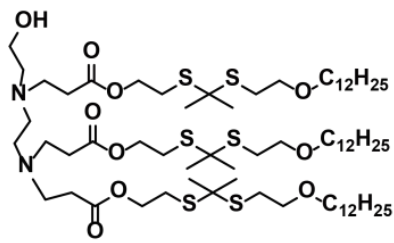

ROS-TK-6

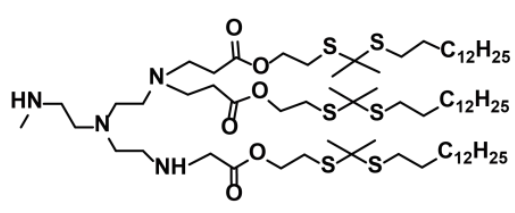

ROS-TK-9

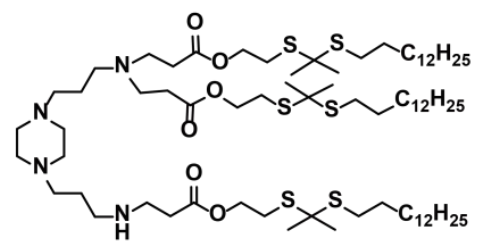

ROS-TK-12

Figure S6 Chemical structure of lipids used for identifying effective RNase A-QPN delivery. 

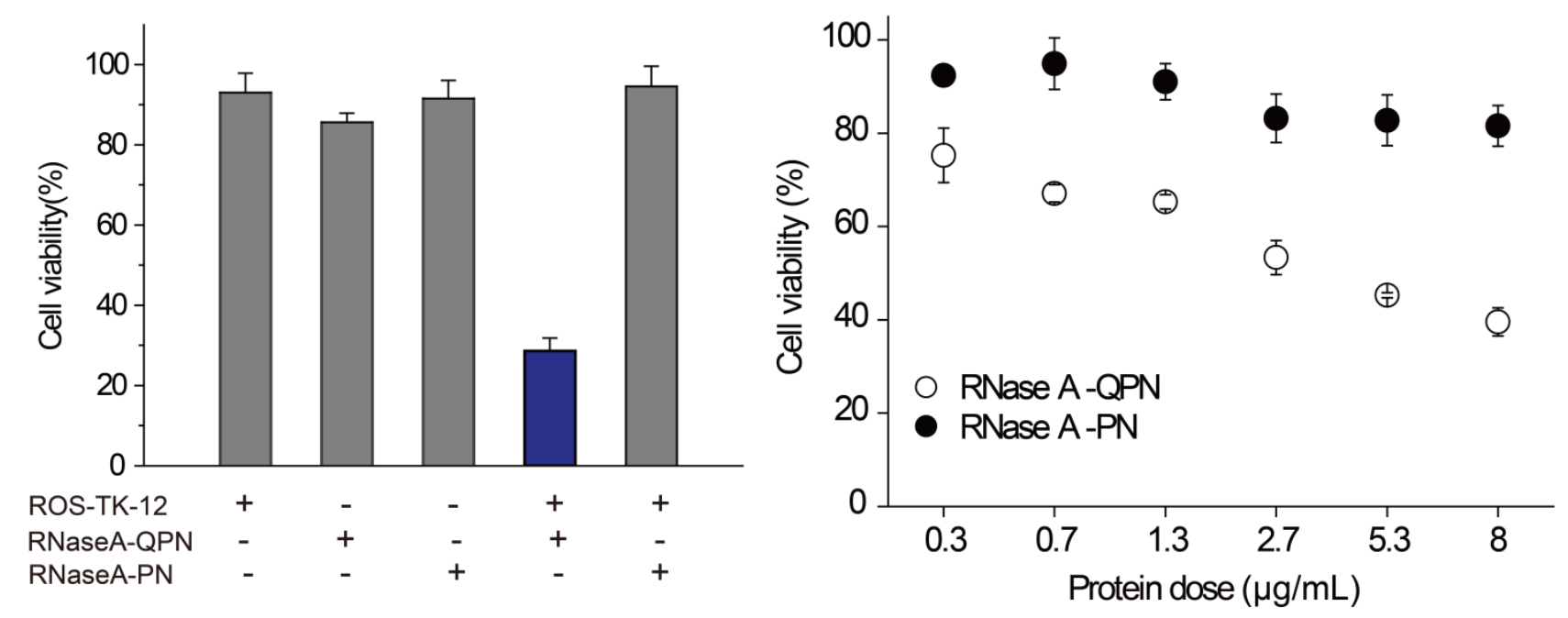

Figure S7 a) Delivery of ROS-TK-12/RNase A-QPN nanoparticles efficiently inhibiting HeLa cell growth. Cells were treated with $8 \mu \mathrm{g} / \mathrm{mL}$ RNase A-QPN or RNase A-PN complexed with ROS-TK-12 (12 $\mu \mathrm{g} / \mathrm{mL}) ; \mathrm{b})$ Protein dose dependent cytotoxicity of RNase A-QPN on HeLa cells. Cells were treated with different concentrations of ROS-TK-12/RNase A-QPN or ROS-TK-12/RNase A-PN nanoparticle at the indicated concentrations. Results are presented as mean \pm SD of three independent experiments. 


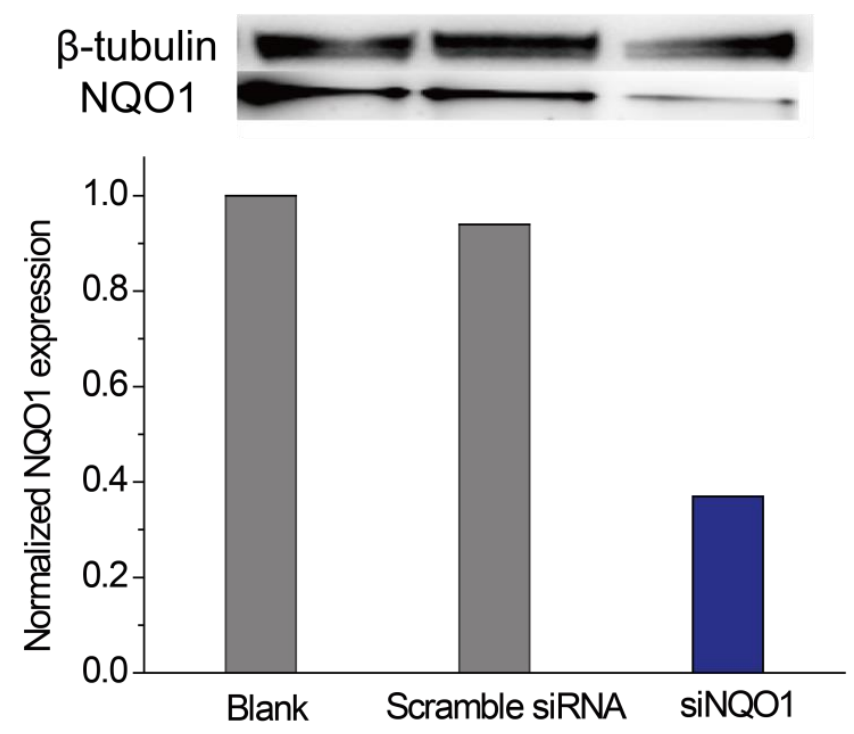

Figure S8 Western blot analysis of HeLa cells treated with siNQO1 (80 nM) or scramble siRNA after transfection using Lipofecatmine 2000. 


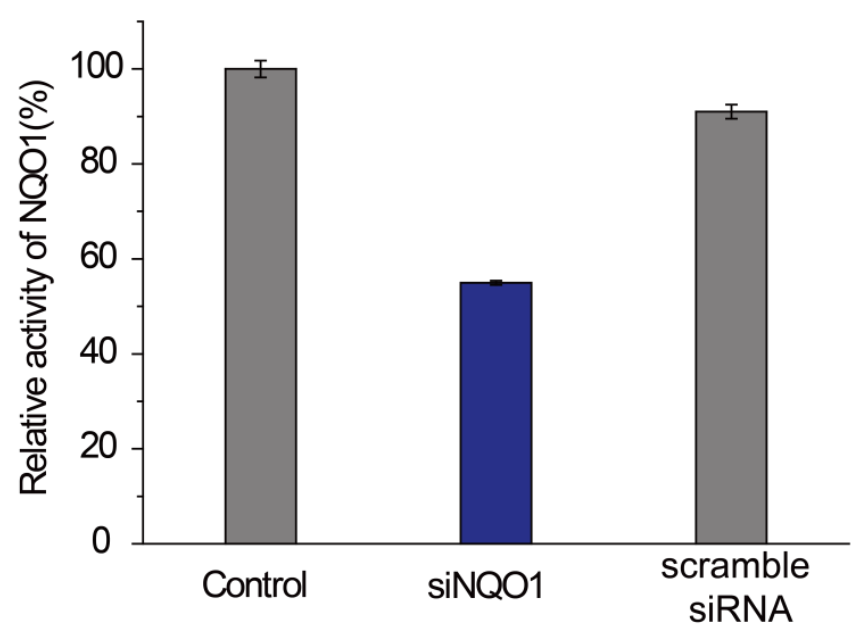

Figure S9 Intracellular level of NQO1 in HeLa cells treated with siNQO1 (80 nM) or scramble siRNA (80 nM) after transfection using Lipofectamine 2000. Results are presented as mean \pm SD of three independent experiments. 


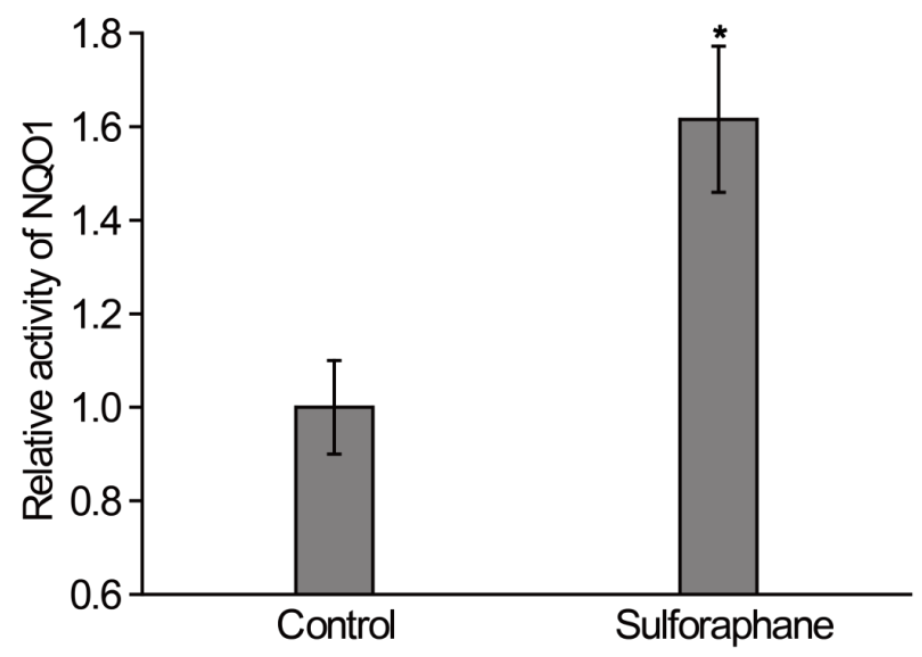

Figure S10 Sulforaphane treatment efficiently enhanced the intracellular NQO1 level in HEK293T cells. HEK cells were pre-treated with $1.33 \mu \mathrm{M}$ sulforaphane for $12 \mathrm{~h}$ before NQO1 activity assay. Results are presented as mean \pm SD of three independent experiments. 


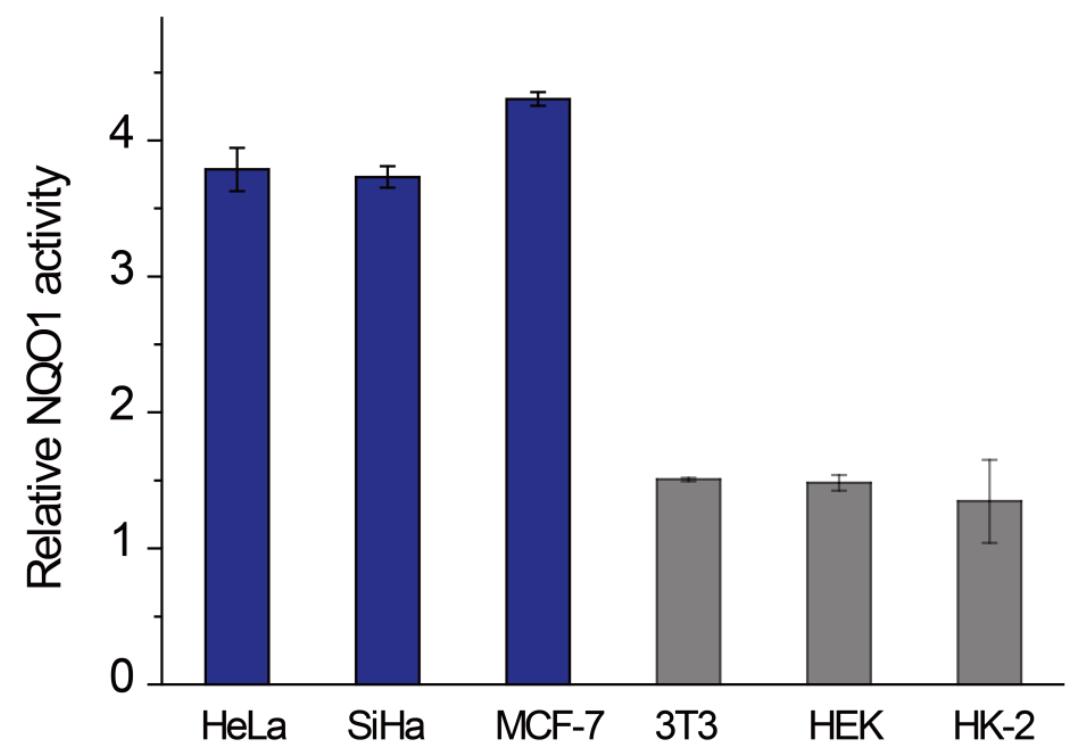

Figure S11 Relative NQO1 activity in cancer cell and non-cancer cell lysates. Results are presented as mean \pm SD of three independent experiments. 


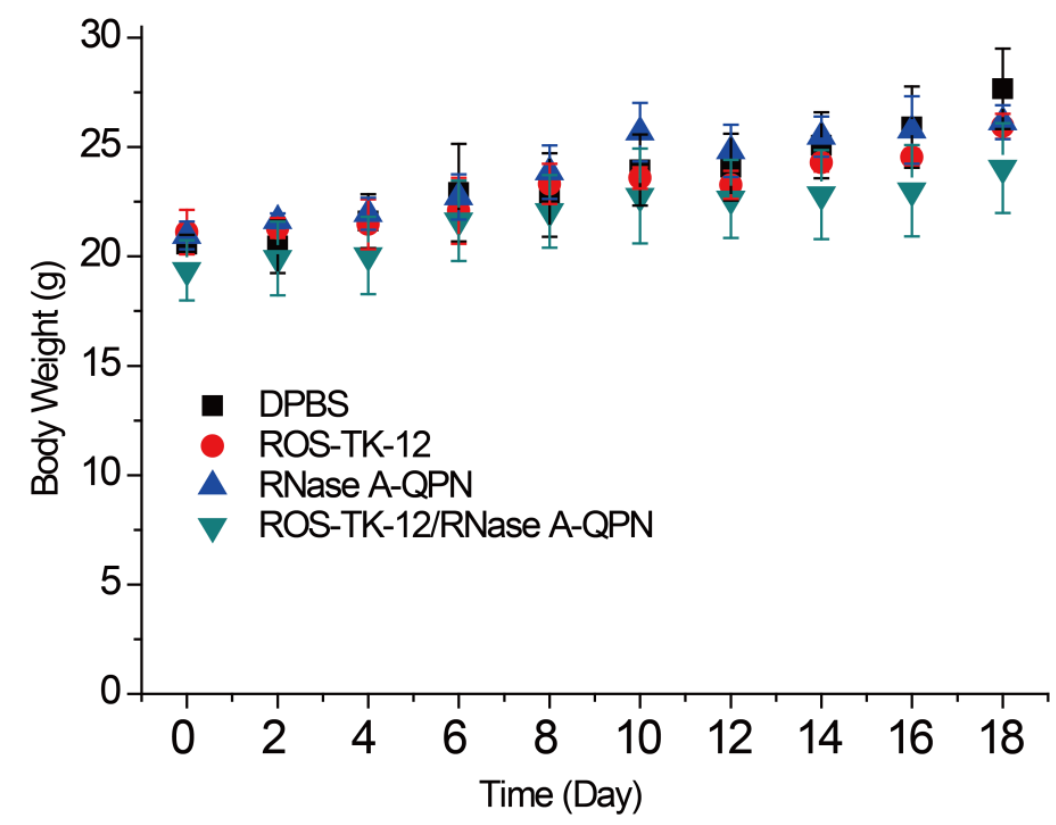

Figure S12 Body weight change of HeLa tumor bearing mice treated with ROS-TK-12/RNase A-QPN nanoparticles, free RNase A-QPN, ROS-TK-12 nanoparticles or DPBS. Results are presented as mean \pm SD ( $n=6$ mice per group).

\section{Reference}

1. Tang, Q.; Wang, J.; Jiang, Y.; Zhang, M.; Chang, J.; Xu, Q.; Mao, L.; Wang, M., Developing chemically modified redox-responsive proteins as smart therapeutics. Chem. Commun. 2019, 55, 5163-5166.

2. Liang, C.; Chang, J.; Jiang, Y.; Liu, J.; Mao, L.; Wang, M., Selective RNA interfe rence and gene silencing using reactive oxygen species-responsive lipid nanoparticles. Chem. Commun. 2019, 55, 8170-8173. 